\title{
Publisher's Note: Phase diagram of interfacial growth modes by vapor deposition and its application for ZnO nanostructures [Phys. Rev. B 96, 115411 (2017)]
}

Da-Jun Shu, Xiang Xiong, Ming Liu, and Mu Wang (Received 12 September 2017; published 18 September 2017)

DOI: 10.1103/PhysRevB.96.119908

This paper was published online on 7 September 2017 with an error in the title of Sec. III on page 2. Section III title should read as "III. CRITICAL ISLAND SEPARATION." The section title has been corrected as of 12 September 2017. The section title is correct in the printed version of the journal. 